\title{
VISUAL STRUCTURE ANALYSIS OF COMPOUND RELIEFS OF MILLI HATS USED BY MEN IN MAKRIYAN REGION
}

\author{
A. A. Yousofkand \\ Master of Visual Communication- Faculty of Arts and Architecture- Tarbiat Modarres \\ University, Iran
}

Published online: 16 July 2016

\begin{abstract}
The major part of Kurdish arts has been traditionally created by women. These women have inherited old distant memories generations after generations and will also leave them as inheritance to succeeding generations. The most significant of these arts is the art of knitting which bears significant importance amongst women. The Milli hat is one of the most elaborately hand-woven crafts created by Kurdish women in Makriyan region. They use diverse ways of symmetry to bring graceful and exquisite reliefs into being and present them to their men. The present study aims to collect, identify and visually analyze the compound recurring reliefs on this type of hat. The research was initiated with the question "What are the visual structure and visual characteristics of compound reliefs applied on Milli hats by men in Makriyan region?". The descriptive-analytical method is used. Therefore, the reliefs were firstly collected and identified based on field-study method, then they were separated and analyzed in terms of visual structure of reliefs. The results showed that most decorative motifs used in this type of hand-woven craft had geometric structure. They were generated using diverse methods of symmetry and created various and beautiful reliefs with live geometric structure. The compound reliefs were mostly created by translational symmetry. The data were collected using field-study method, written sources, library as well as a global information network (Internet).
\end{abstract}

Author Correspondence, e-mail: aso.azizi@yahoo.com

doi: http://dx.doi.org/10.4314/jfas.v8i2s.121 
Key Words: traditional arts, Milli hats by Makriyan, compound reliefs, symmetry

\section{INTRODUCTION}

In traditional arts, the hand-made and woven crafts by rural population far from urbanization and distant from characteristics and beliefs of urban societies who are not familiar with other civilizations hold special place since what they offer comes from the collective unconscious rooted in their ancient art and culture which were inherited from their predecessors and carry the least impact of urban environment. The most striking feature of these art is their practical aspect which has been transformed to their needs with the passage of time and experienced changes in structure and semantics with further usages of new materials. The artists of the old periods were used to extract the raw materials from nature and imagined themselves part of the nature when they were creating artistic pieces. In these civilizations, the artist presented ethnic views as symbols and motifs representative of the period, however the meaning and structure of these reliefs were gradually forgotten as though contemporary human has weak relation to them. Therefore, the necessity of collecting, remembering and recognizing motifs used in traditional arts is more evident.

The hand-woven crafts, due to their bonding with public life, variety and abundant designs and ornaments contain more of the history and adventures of a community (Jazayeri, 1991:5). One of the most important and significant traditional arts in Makriyan region is the weaving art with high number of admirers amongst women. With regard to the type of craft and peoples' need, diverse instruments were used such as hat, socks, glove, clothes, curtain, carpet, Give, etc. Each of them is woven using special tools and materials and so they differ in their decorative reliefs. Among the handicrafts made by Makriyan women, an elaborate type of hat woven by mill (weaving hook) attracts attention and so is called "Milli hat" in the region. It is sometimes called "Mirzaei hat". This type of hat is decorated with various single and compound designs which are regarded as important visual sources of this region. The single reliefs could be semantically categorized in "plants, animals, abstract, mythical and inspired by other phenomena" groups. The compound group was created by a combination of all categories of single groups. The transitional, reflexive, rotational and compound symmetry were also applied. The translational symmetry method mostly uses the abstract single reliefs. The compound reliefs generally use the animal single reliefs such as pigeon.The researcher has collected the reliefs on Milli hats in 
Makriyan region mostly used by rural men and analyzed the complication of destruction and oblivion of this art although it dates back thousands of years and its live visual structure signifies the well culture and civilization of the peoples.

\section{Review of Literature}

Among analytical writings on the role of motifs, ornaments of traditional arts and handicrafts in Iranian art, certain subjects on well-known civilization have often been worked on and little fundamental researches have been devoted to ethnic art. This is a pioneer researcher which collected and analyzed data on visual structure and description of reliefs in Milli hats used by men in Makriyan region. The pictures were selected mostly by field-study method from Abdollah Samadi archive and were redesigned and reconstructed by researcher.

\section{Methodology}

The study is conducted based on an analytic-descriptive method. The study aims to discover the facts and realities and recognize the motifs on Milli hats used by men in Makriyan region. It also discusses the visual structure of these motifs. The reliefs were firstly collected and eventually analyzed in terms of visual structure. The data were collected using field-study method, written sources, library as well as a global information network (Internet).

\section{1- Milli Hats made by Makriyan people}

Each artist speaks the language of his ancestors (Hawers, 2003:48). The beliefs and traditions proceeding from generation to generation with little changes caused the artists to remain faithful to his past ethnic and collective memories and use these motifs in his literary works with the least possible change in form and content. Dr. Shaygan says: "One of the indicating aspects of East Art is its loyalty to collective memory. It is in fact a memory constantly preserving the mental structures and general cultural system. In this system, the forms are revitalizing not only in their external manifestation but also in their essence". The example of this fact is that Asian artists have been repeating the same pattern enriched with their inner experiences. The artist does not much intend to flaunt for his personal invention but tries to be faithful to memory and revive its treasures. The memory makes a union between past, present and future and flourishes all on the timeless horizon of the moment (Shaygan, 1992: 261).

Although we simply cannot find the traces of painting and visual art just as Classical Europe and Traditional Iranian Arts in the background of this historical civilization, due to the historical circumstances and social area of Kurdish people, however we can claim that the motifs used in 
traditional arts of these people are a sort of visual art of this tribe. The Kurdish art is full of sincerity and love to country which brings us to a distant past and divulge many of the untold facts of history.

In one corner of the vast territory of northern West Iran, there is an area with towering mountains, deep cliffs and pleasing springs known as Makry Kurdistan centralized by Mahabad town (Rozbiyani, 2006:45). The Makriyan Kurds mostly apply geometric motifs in their works which clearly exhibit their inspiration from nature. The weavers masterfully reflect natural beauties and variety of plants and animals (Moshtagh, 1995:122). The most significant art of Makriyan people is the art of knitting with abundant admirers amongst women. With regard to the type of craft and peoples' need, diverse instruments were used such as hat, socks, glove, clothes, curtain, carpet, Give, etc. Each of them is woven using special tools and materials and so they differ in their decorative reliefs

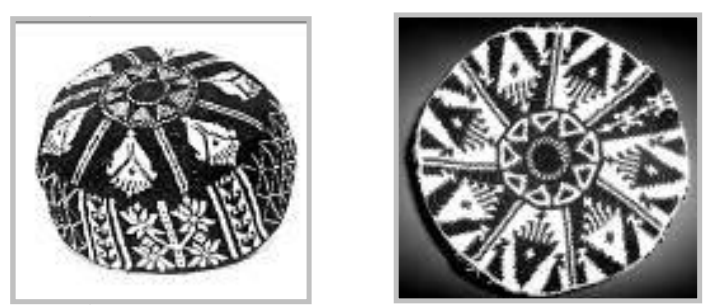

Fig.1. Milli Hat made by Makriyan people

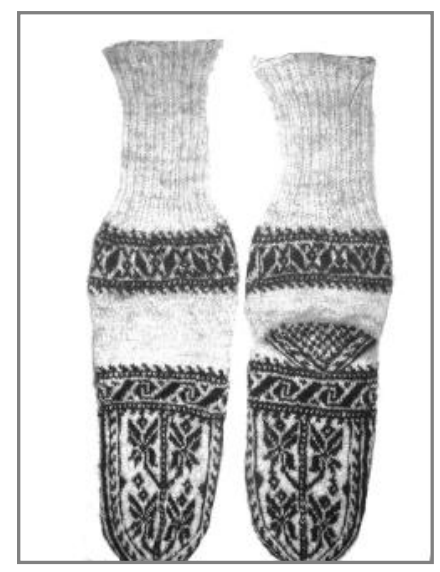

Fig.2. Designs and ornaments on Makriyan wool socks; Source: writer 
Among the handicrafts made by Makriyan women, an elaborate type of hat woven by mill (weaving hook) attracts attention and so is called "Milli hat" in the region. It is sometimes called "Mirzaei hat". The hat decorated with decorative designs is used by men (Figure 1). This type of hat has a very fine and delicate texture which is prepared in three colors of white, black and white as well as colored. Mirzaei hat or Milli hat has different textures and decorative motifs with regard to its usage. They are categorized on the basis of color as Milli hat, Shakaki hat, Wire hat, white hat, Ghermeh hat etc. and shown in table 1. Each category of these hat possess distinctive type of form and structure. Since the present paper is not extensive enough to examine each category individually, it focused on the collection and analysis of visual structure of common compound decorative designs which hold the least possible difference.

Table 1. Types of Milli hat and color variety; Source: writer

\begin{tabular}{|c|c|c|c|c|}
\hline Colored & $\begin{array}{c}\text { Black \& } \\
\text { White }\end{array}$ & White & $\begin{array}{c}\text { Type of } \\
\text { Hat }\end{array}$ & Row \\
\hline$*$ & $*$ & $*$ & Milli hat & 1 \\
\hline$*$ & $*$ & $*$ & $\begin{array}{c}\text { Shakaki } \\
\text { hat }\end{array}$ & 2 \\
\hline & $*$ & $*$ & $\begin{array}{c}\text { Mantle } \\
\text { hat }\end{array}$ & 3 \\
\hline$*$ & $*$ & $*$ & $\begin{array}{c}\text { Ghermeh } \\
\text { hat } \\
\text { Etc. }\end{array}$ & 6 \\
\hline
\end{tabular}

\section{2- Designs and Ornaments on Makriyan Milli Hats}

One of the main characteristic of decorative designs used in a variety of traditional handmade crafts is their subjectivity known as "mental-woven" (Sharifzade, 2002: 38). The weaver is a Kurdish woman who draws beautiful, diverse and dynamic geometric motifs in a perfect geometric manner relying on her imagination based on the hat usage.

The vertical, horizontal, slant and cursive lines as well as metaphor and crypto processing are amongst the common ways to prepare these designs. Milli hat, similar to other traditional handicrafts, is a useful craft used by men in different styles along with colored turban to protect them from heat and cold. It is to be mentioned that Milli hat is alone put on with no turban in hot weather of summer. 
One of the important tasks of decorative motifs on Milli hat is to balance the environment coarseness and subtilize the everyday life. These designs and motifs are completely presented geometrically and no curved line is observed. The type of applied instrument dose not enable the weaver to make the designs by curved and round lines. Multiple knots in denticulate form as neighboring points made with small hooks offer the weaver the opportunity to create only a limited number of designs. So, the weaver is deprived of making other designs and adjust the structural form of many possible motifs to her weaving tools. However, other handicrafts which make use of materials and instruments different from Milli hat exhibit simpler and more extensive designs and create less restrictions compared to Milli hats.

The extensive designs used in Milli hats presented in Table 2 are categorized into "human, animals, abstract, mythical and inspired by other natural phenomena" groups. Each group contains the samples designs used in Makriyan Milli hats and their frequency is also mentioned according to the related type and group. From the total 85 single designs collected for further study, 17 samples are demonstrated in Table 2.

Table 2. Frequency of decorative motifs on Makriyan Milli hat, Source: writer

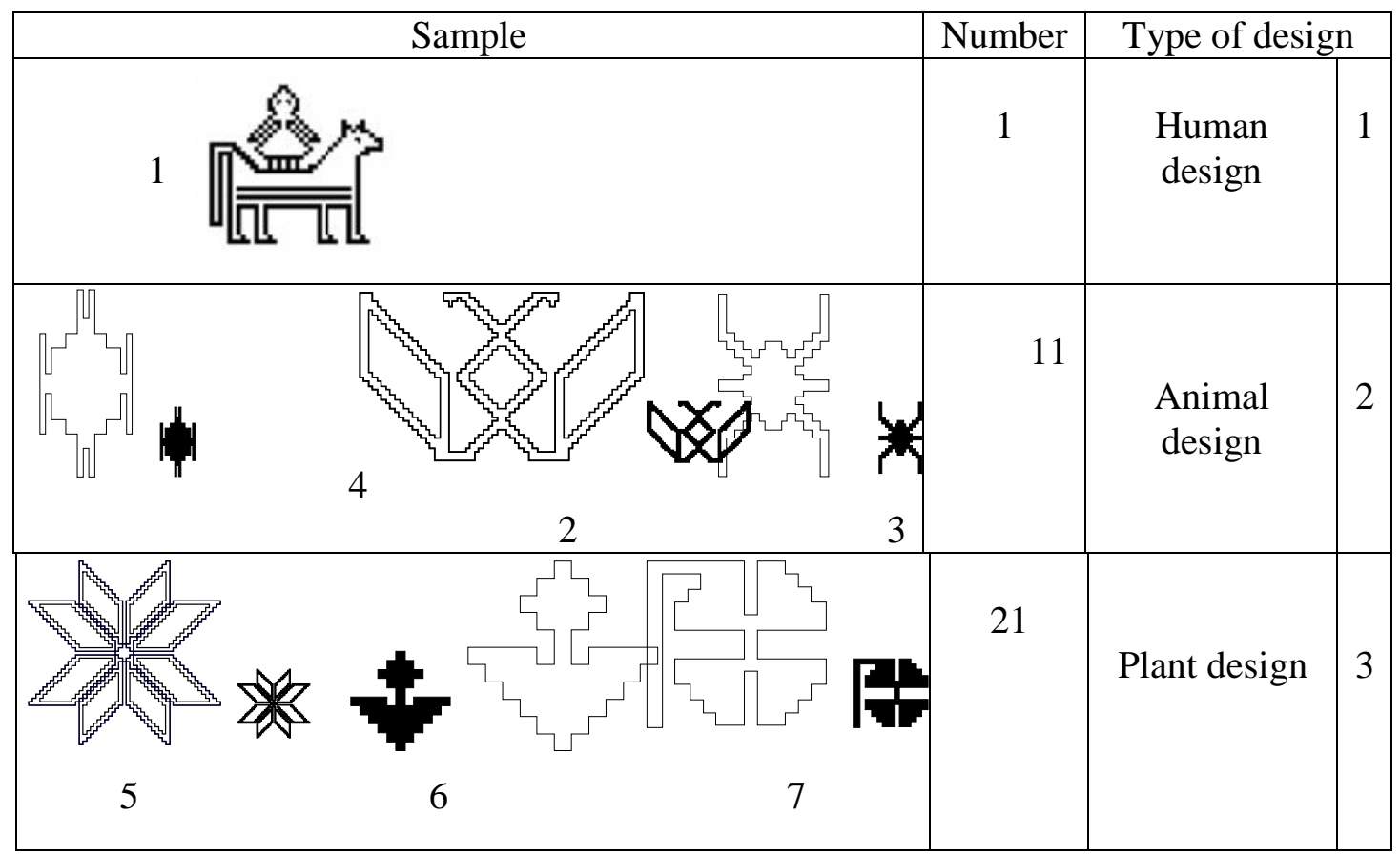




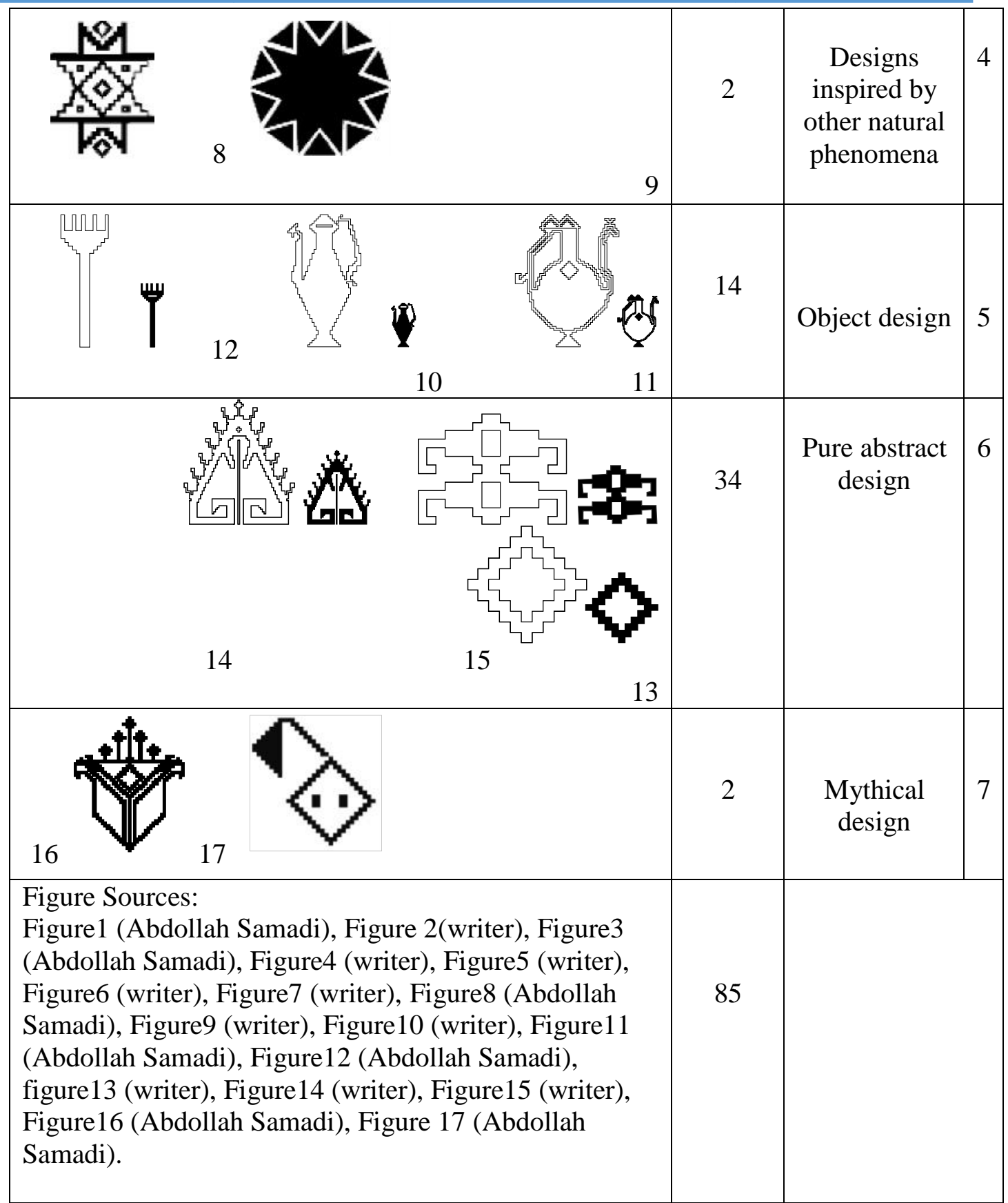




\section{3- Structure of Milli hat}

Milli hat has two structurally distinct parts. The upper part of hat is usually woven circularly. Then the margin is woven in rectangular form and finally the two parts will be sewn together. Although these two parts have decorative motifs with sharp differences, however they use the same way of weaving, tools and materials.

\section{1-3- Decorative Designs on Milli Hat}

The upper part of Milli hat is woven circularly as much as the size of user's head. The weaving is usually initiated from the central part of top circle and in most case ends in the shape of a sun with small rays. A beautiful abstract compound design on the margin usually cover the whole circle which is created by symmetry method and separate the upper part from margins and main body of hat. Then the weaver fills the empty space usually with plant designs or leave them white and empty. The plant designs and motifs taken from other phenomena are generally responsible to decorate this part of hat and other designs are less common. More precise examination of mythical role of certain motifs derived from celestial bodies like the sun and stars used in upper part of hat indicated that they hold the concept of cosmic protection of user and ward off evil. The mythical motifs are often created using rotational symmetry method (Figure3).
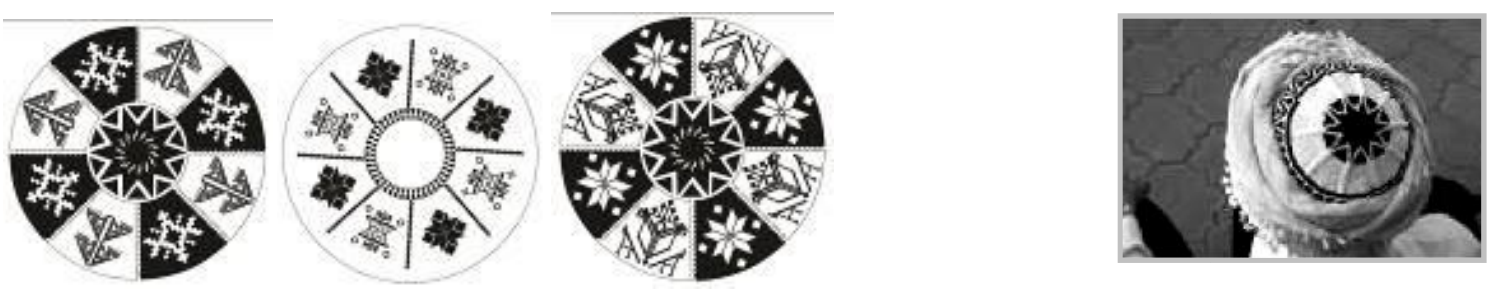

Fig.3. Milli hat designs; source: writer

\section{2-3 Marginal Designs on Milli Hat}

The margin and main body of hat is woven as rectangle in the size as much as the user head. The weaver divides this space into four or eight parts by compound and recurring designs. She also fills the inner parts by other designs in different size. The common practice is to weave one of the plant or animal designs in the maximum possible size and fill the marginal spaces with other 
designs in smaller size (Figure 4). The animal designs are more common in marginal part of hat than others, even though other designs will be delineated with no limitation. However this part of hat is mostly decorated with compound reliefs.

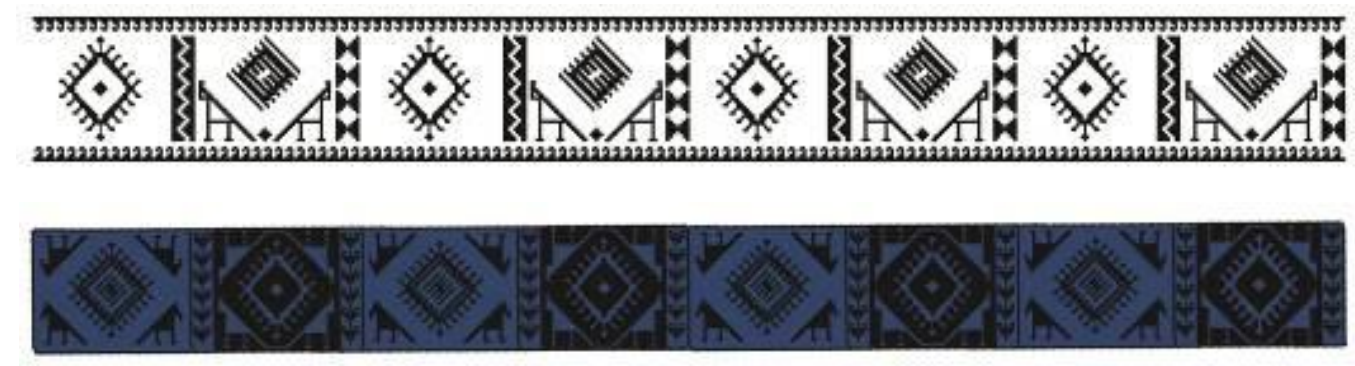

Fig.4. Marginal reliefs on Milli hat; Source: writer

\section{4- Generation Methods of Reliefs on Space}

The tendency to symmetry, harmony and regular phenomena is one of the most constant aspects of human civilization. Human believe in the coincidence and symmetry between phenomena and his effort to achieve symmetrical and harmonious existence appears in all fields of knowledge, art and industry. In addition to physiological factors, material factors and physical phenomena in surrounding environment play significant role in human consciousness to these features and their uses. Symmetrical, analogous, regular and coordinated phenomena in nature such as sequential order of the day and night, the seasons of the year, etc. attract the human attention to existing harmony. Symmetry found in many natural phenomena, animals and humans has been existing from the beginning of creation (Sharif Zade, 2002: 22).

The artists generally search for creating beautiful and proportional combination which signifies the whole-to-part relation in an artistic work. The parts are also in relation and coordination with each other as well as the whole. In many cases, the artist makes compound reliefs to create harmony and balance. The artist geometrically reduplicates the design and expand it at a broad space. Architectural decorations are instances of such designs which present beauty through recurring designs and symmetry (Sharif Zade, 2002: 22).

There are various ways to reduplicate and develop the reliefs on the same level. In symmetry and design expansion, the reliefs are sometimes changed while they are fixed in some other cases. 
The symmetry methods could be divided into four distinctive categories: translational, reflexive, rotational and compound symmetry (Sharif Zade, 2002:23).

\section{1-4- Translational Symmetry}

Translational symmetry is the oldest and simplest method to replicate the reliefs on a space. There are numerous instances of literary works in Ancient Iranian and Islamic History which were created with simple repetition of a relief or design. This simple method which is able to create astoundingly beautiful artistic pieces could be seen in architecture of Achaemenid and Sassanian Empire palaces and reliefs on Silak, Marlik and Giyan clay dishes. In this method, the design does not change. The new designs will be actually created by changes in specified size and direction which render a new location and so a new design (Sharif Zade, 2002: 24).

A Makriyan female artist consciously uses this method in most of her artistic works (Figure 5) as though we can claim that this type of delineating is observed in majority of traditional handicrafts. However the most elegant of these decorations belongs to Mill hat designs. The Makriyan Kurdish women have properly used positive and negative spaces and created unique works using translational symmetry method and replication of reliefs on Milli hats. The artist benefits from beautiful colors, calculates the outer space of hat and makes the most proper divisions. This method uses different types of plant, abstract and animal reliefs to create new designs. The abstract and animal images, pigeon specially are the most common reliefs used on the hat margin to create compound designs.

\section{1 \\ 난ㄷㄴㄷㄴㄷㅗ}

Fig.5. Abstract compound design by translational symmetry method; source: writer

\section{2-4 Axial or Reflexive Symmetry}

The reflexive methods possesses more complexity and specific features compared to translational method. In this method, the intended relief or design repeats around an axis in reverse similar to what is reflected in a mirror image. The traditional artists called this method "pivotal symmetry". In this method, the two forms are identical and/ or similar but not 
conformed unless we turn one of the reliefs. This method, just as other methods, has its own characteristics and special delicacies and Old Iranian artists have applied it to present the most precious artistic works in different areas. The most prominent instances of this method are mosque entrances and minarets.

Makriyan female artists consciously used this method in most of her artistic works (Figure 6) as though we can claim that this type of delineating is observed in majority of traditional handicrafts. The artists have properly used simplified animal reliefs on the margins to create unique works. The use of plant designs has also intensified the design's beauty in some cases. The proper use of positive and negative spaces has increased its artistic attraction and capability. The plant images in the middle of two animals, pigeon specially are the reminiscent of life tree in Ancient Iranian artistic works.
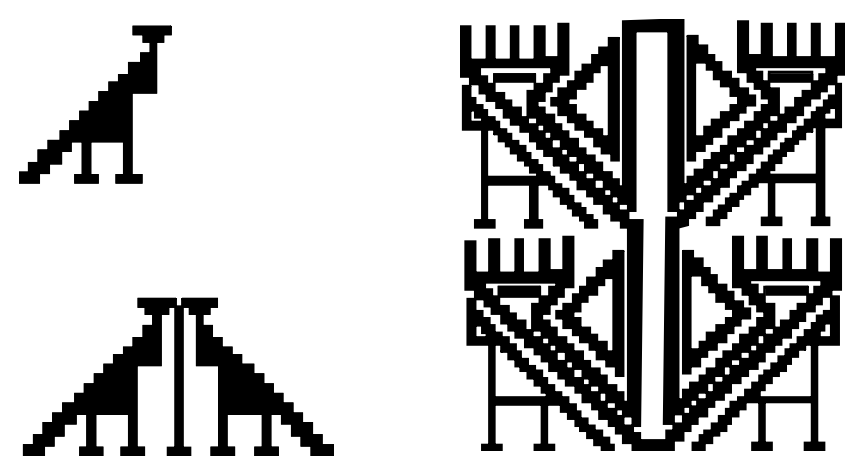

Fig.6. Compound designs with pigeon relief by reflexive symmetry method: source: writer

\section{3-4- Rotational Symmetry}

This type of symmetry is more complicated and so attractive than previous methods. In this type of symmetry, a relief is repeated around a single point as much as it covers the whole circle. Therefore we could make a design which is repeated (reflected) around a common axis. It is also possible to rotate a relief around a single point with specified angle. In both methods, the design should cover the whole circle (Sharif Zade, 2002:28).

There are abundant instances of rotational symmetry in Ancient Iranian art in different periods as though many of the beautiful designs on Old Iranian clay dishes were created by this method. However the most perfect and attractive designs were created in Islamic period using rotational symmetry method. The apogee of using this method is observed in plates remained from Islamic 
period. This method was also applied in architectural works e.g. mosque domes made in circular form or four arcades mosques with $90 \mathrm{C}$ rotational symmetry.

In Makriyan hat, this method is mostly used to design the upper and central part of hat which is inspired by monotheism and is always initiated from and point to a single point. In other words, the upper part of Milli hat is formed as a circle which is the main point of decoration and fills the hat space with rotational method (Figure 7). In most cases, the design is inspired by sun which protects the user from cold and hot weather by its cosmic heat and power.

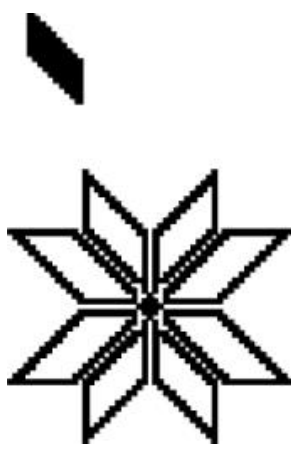

Fig.7. Compound design of Garchak flower by rotational method; source: writer

\section{4-4- Compound Symmetry}

In this type of symmetry, the characteristics of translational, reflexive and rotational symmetry offer the compound symmetry. This type of symmetry is mostly used in rotational symmetry generated from reflexive or translational symmetry and evolves into rotational type (Shari Zade, 2002:31).

The Kurdish weaver who is perfectly aware of these methods creates beautiful designs. In Milli hats, major part of design is expanded in marginal spaces. The artists uses all her techniques and skill to create new spaces and avoid repetition and exaggeration as much as possible (Figure 8).

The noteworthy point in the context of symmetry is to create asymmetry in symmetry. Looking at nature, we will see that in all symmetrical designs and reliefs, there are also asymmetrical points. This is the point which makes a perfect and attractive symmetrical work. For example the human face has reflexive symmetry. The axis passing through the nose divides the face into two parts and so it exhibits a sort of harmony and beauty. To be more precise, there are slight points 
in two half of the face which are different and asymmetric. There are maybe a spot or wrinkle in one half of the face which is absent in other half and so make the symmetry of the face more beautiful (Sharif Zade, 2002:31).

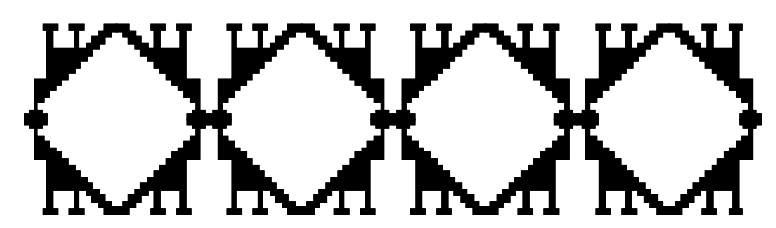

Fig.8. Compound pigeon designs by compound method; source: writer

\section{CONCLUSION}

The people in Makriyan region, just like to other scattered tribes and civilizations of Iran and the world, have their exclusive culture and art. Although we simply cannot find the traces of painting and visual art just as Classical Europe and Traditional Iranian Arts in the background of this historical civilization, due to the historical circumstances and social area of Kurdish people, however we can claim that the motifs used in traditional arts of these people are a sort of visual art of this tribe. The Kurdish art is full of sincerity and love to country which brings us to a distant past and divulge many of the untold facts of history. Finally the compound designs on Milli hats in Makriyan area could be summarized as follows to answer the research questions: Amongst the 85 single designs, 34 abstract designs had the highest rate of application on Milli hats while human reliefs showed the least rate. The decorative designs were not used just as ornaments but they narrate the story of human needs and beliefs. They are sometimes used to make a more delicate environment and adjusted the violence of surrounding environment. The Kurdis female artist is aware of different symmetry methods in traditional art and benefits from common ways of rotational, reflexive, and compound symmetry methods. The dominant reliefs in rotational symmetry are mainly single abstract reliefs. The compound symmetry method mostly apply single animal reliefs such as pigeon. The compound designs created by rotational symmetry are generally responsible for creating separate spaces for more dominant reliefs. However in some cases, the compound reliefs made by rotational symmetry leads to the creation of the most important and prominent relief, in terms of status and position, such as sun cross on Milli hats. These designs are created geometrically and the limited instruments prevent the weaver to create many of the designs embodied in traditional regional arts. In other words, there 
are only limited number of designs belonging to Kurdish tribe on Milli hats. The restriction of instruments used to weave Milli hats makes broken and denticulate lines present while the curved lines are not often seen on these hats. But it is not true for other traditional handicraft arts in this region. The Kurdish female weaver creates diverse live designs with no plan prepared in advance relying on their own imagination and the hat uses. This type of weaving is known as "mental-woven" in traditional arts.

\section{REFERENCES}

- Hosseini, M, “Traditional Design Workshop”, Textbook Publishing Co.: Tehran, 2008.

- Jazayeri, Z, “Introduction to Kilim”, Soroush Pub.: Tehran, 1991.

- Roojbiyani, M. J. "Lords of Makriyan”, Ana Pub. : Tehran, 2006.

- Shaygan, D, “Asia against West”, Bagh e-Ayeneh Pub.: Tehran. 1992

How to cite this article:

Yousofkand A A. Visual structure analysis of compound reliefs of milli hats used by men in makriyan region. J. Fundam. Appl. Sci., 2016, 8(2S), 1798-1811. 